\title{
CAPITAL SOCIAL COMUNITARIO Y PROCESOS INSTITUCIONALES Barrio Santa Clara, Municipio Distrito Central, Honduras*
}

\author{
Community Social Capital and Institutional Processes \\ Barrio Santa Clara, Municipio Distrito Central, Honduras
}

\author{
Ayes Rivera, Ixchel \\ iayes@uc.cl
}

\section{RESUMEN}

En un contexto de informalidad, el Capital Social Comunitario (CSC) es un factor determinante para que las comunidades de los barrios informales logren satisfacer sus necesidades básicas y sean reconocidos por las instituciones prestadoras de servicios y administradoras del territorio municipal. Esta investigación se concentra en las dinámicas sociales y en las redes internas del barrio Santa Clara a través de un estudio exploratorio y descriptivo con metodología mixta, que permitió describir la situación socio-económica de la comunidad, dimensionar el CSC a través de sus recursos asociativos (confianza, reciprocidad y cooperación) y analizar la situación sobre la tenencia de la tierra, vivienda y acceso a servicios urbanos, señalando la incidencia que tienen las instituciones externas en la organización comunitaria y en los procesos de consolidación barrial.

Palabras clave: Capital Social Comunitario, Consolidación Barrial, Institucionalidad Bloque temático: La ciudad Poscrisis. Transformaciones socio-urbanas y políticas.

\section{ABSTRACT}

In a context of informality, the Community Social Capital (CSC) is a determining factor for informal neighborhood communities to satisfy their basic needs and be recognized by the public service providers and the municipal territory managers. This research focuses on the social dynamics and internal networks of the Santa Clara neighborhood through an exploratory and descriptive study with a mixed methodology, which allowed describing the socioeconomic situation of the community, dimensioning the CSC through its associative resources (trust, reciprocity and cooperation) and analyze the situation of land tenure, housing and access to urban services, noticing the influence that external institutions have on the community organization an neighborhood consolidation processes.

Keywords: Community Social Capital, Neighborhood Consolidation, Intitutionality

Topic: The post-crisis city. Socio-urban an political transformations.

\footnotetext{
* Tesis presentada en el Instituto de Estudios Urbanos y Territoriales (IEUT) de la Pontificia Universidad Católica de Chile (UC), realizada con el apoyo del FONDECYT regular 1161550 "Densidad residencial y redes de sociabilidad. ¿Paradojas de la metropolización? a cargo del investigador Felipe Link.
} 


\section{Introducción}

Las dificultades que atraviesa la población en condiciones de pobreza para poder adquirir un espacio habitable en la ciudad se encuentran relacionadas al modo en que la vivienda es vista como una mercancía. Sus posibilidades se reducen a aquellas que puedan encontrar en la ciudad informal, con maneras de construir, hacer y habitar la ciudad propias de las "lógicas de apropiación y ordenamiento que determinan su identidad" (Pino y Ojeda, 2013: 119).

Existe evidencia empírica en la región latinoamericana que relaciona el modo informal de habitar la ciudad con barrios en donde se "generan prácticas (...) que toman como apuesta principal su disponibilidad de capital social" (Gutiérrez, 2008: 6). El capital social está relacionado a los elementos y situaciones socioculturales comunes de un grupo (identidad, religión, amistad, parentesco, etc.) (Tironi, 2003), y en el caso de los barrios informales, las condiciones precarias y la necesidad de un espacio para habitar hace que las personas, independientemente de su origen, compartan intereses y ambiciones.

Por otro lado, las acciones de instituciones gubernamentales y de otros agentes externos, suelen ser factores importantes para la consolidación de este tipo de barrios. Es común que las instituciones prestadoras de servicios asuman la responsabilidad de transferir el bien y de dar mantenimiento a la infraestructura que los pobladores logran instalar en sus barrios con esfuerzos propios (Pírez, 2013), reconociendo así sus derechos y legitimando los barrios de origen informal.

Esta investigación se basó en los antecedentes de trabajo conjunto de la comunidad del barrio Santa Clara con instituciones gubernamentales y la ONG TECHO-Honduras. Parte de la hipótesis de que la comunidad cuenta con un Capital Social Comunitario (CSC) que les permite desarrollar características propicias para alcanzar exitosamente el cumplimiento de algunas gestiones relacionadas a recursos y apoyo técnico necesario para concretar proyectos de infraestructura que contribuyen a la consolidación barrial.

El objetivo general ha sido comprender cómo convergen el CSC y los procesos institucionales y cómo dicha relación incide en la consolidación barrial. Para alcanzar este objetivo se debieron reconocer los diferentes factores e instituciones que inciden sobre el territorio, caracterizar los recursos asociativos que configuran el capital social comunitario en las esferas de socialización internas de la comunidad y entender la relación de los procesos institucionales con los modos de organización comunitaria y los procesos de consolidación barrial.

Este es un estudio exploratorio y descriptivo con metodología mixta, cuya parte cualitativa dio lugar a la descripción, análisis e interpretaciones de la realidad social de la comunidad y de las instituciones estudiadas. Algunos elementos y prácticas relacionadas a los recursos asociativos del CSC fueron medidos e interpretados a partir de preguntas de carácter comparativo y calificativo que formaban parte de una encuesta aplicada a 119 participantes, misma que permitió recabar información en tres dimensiones: 1. Situación socio-económica de la comunidad; 2. Categorías de confianza, reciprocidad y cooperación, y 3. Información sobre la tenencia de tierra, vivienda y acceso a servicios urbanos. En paralelo se realizaron entrevistas semi-estructuradas a seis representantes institucionales y dos referentes comunitarios. 


\title{
2. Marco conceptual
}

\begin{abstract}
"Los pobladores y colonos son una fracción de la población urbana que está obligada a vivir en condiciones de extrema precariedad física como consecuencia de un doble conjunto de contradicciones: el desarrollo desigual de la urbanización y de la productividad económica, y la incapacidad tanto del capital privado como de las agencias gubernamentales para proporcionar los servicios urbanos requeridos para la expansión de las necesidades colectivas de consumo, en condiciones tales que los hagan asequibles a los nuevos habitantes de la ciudad." (Castells, 1987: 15)
\end{abstract}

Ante el escenario aún vigente planteado por Castells, existen mercados informales a los que recurre la población urbana más empobrecida. La aparición de los mercados informales de suelo puede ser explicada por la existencia conjunta de tres situaciones: 1. La falta de tierra disponible para ocupar; 2. La movilidad residencial, motivada por el "costo de oportunidad" y la "flexibilidad" que ofrece este tipo de mercado y 3 . Un proceso general de empobrecimiento. A pesar de existir transacciones económicas, estos mercados están "mediado[s] por relaciones sociales que no son las típicas del mercado formal, en función de los agentes que intervienen (...)" (Cravino, 2006: 145).

Para esta investigación interesan esas relaciones sociales y se entiende que todo Capital Social requiere de esfuerzos de sociabilidad que le permita reproducirse en un grupo, así como de "una serie continua de intercambios en el cual el reconocimiento es infinitamente afirmado y reafirmado" (Bourdieu, 1986: 8). Para Durston (1999: 104) "el capital social comunitario [CSC] está constituido por normas, prácticas y relaciones interpersonales existentes y observables" a través de tres recursos asociativos que permite dimensionarlo; la confianza, la cooperación y la reciprocidad (Durston, 2003; Atria, 2003).

La confianza es entendida como una "variable psicosocial dinámica, que mide la capacidad y voluntad de dos contrayentes para intercambiar favores e información" (A. de Lomnitz, 1975: 209). La confianza es crucial para que existan intercambios en las redes comunitarias y se desarrollen procesos colectivos.

La cooperación se define como una acción que busca alcanzar objetivos compartidos de emprendimientos comunes (Durston, 2000). Si bien la participación es un modo de visibilizar la cooperación entre vecinos y se encuentra relacionada con el CSC, se debe aclarar que, no por sí misma constituye un capital social, ya que pueden existir intereses individuales que motiven la participación en acciones colectivas (Raczynsky \& Serrano, 2005).

La reciprocidad, para Atria (2003: 583) es un "principio rector de una lógica de interacción ajena a la lógica del mercado, misma que involucra intercambios basados en obsequios". La intensidad con la que se realizan intercambios, el tipo de intercambios, la frecuencia de los mismos, y las condiciones (implícitas o no) de los intercambios realizados dentro del grupo social, son elementos centrales para determinar cómo se manifiesta la reciprocidad en el CSC.

El estudio de estos tres recursos permite reconocer el estado del CSC de una comunidad y determinar su incidencia en los logros comunitarios relacionados a la consolidación barrial. Esta consolidación barrial va más allá de la titularidad sobre el suelo, pues también involucra el acceso a servicios básicos que buscan las comunidades para establecerse en el territorio. Sin embargo, el hecho de contar con la infraestructura que sustente los servicios urbanos no asegura el goce de los mismos, debido a que la conexión a las redes formales depende en mayor medida de la voluntad y la capacidad de las empresas encargadas de distribuir los bienes. Actualmente estos servicios urbanos son adquiridos bajo la lógica de mercado; "son mercancías que deben ser adquiridas intercambiando cantidades de dinero suficientes para garantizar a sus productores la rentabilidad de las operaciones" (Pírez, 2013: 461). 


\section{Presentación del caso}

\section{$3.1 \quad$ Contexto municipal}

El Municipio Distrito Central (MDC), capital de Honduras, se conforma por las ciudades de Tegucigalpa y Comayagüela, separadas por el Río Choluteca. El MDC es la segunda área urbana más grande del país y gran parte de ella es producto de la autoconstrucción de vivienda (Caballero, 2010).

Según datos del Instituto Nacional de Estadística (INE) para el 2016, el 60.9\% del total de la población se encontraba viviendo en situación de pobreza, y el 38.4\% en condiciones de extrema pobreza. Sumado a esto, el país cuenta con una alta desigualdad ${ }^{1}$ y los pobladores de las áreas urbanas viven en graves condiciones de violencia ${ }^{2}$. Todo esto hace que el derecho a la ciudad se convierta en "meras proposiciones teóricas" y que los beneficios urbanos a los que muchos aspiran sean muy limitados (Trundle, 2012).

\subsection{Caso de Estudio}

El barrio Santa Clara se encuentra en un cerro ubicado en el sector noroccidental de la ciudad de Comayagüela. Este sector es un área periurbana y forma parte del tejido urbano por las manifestaciones propias de la ciudad que predominan sobre las características del campo (Lefebvre, 1970). Este barrio se encuentra dentro de la sub-cuenca del Río Guacerique, la cual fue delimitada bajo acuerdo No. 03-73 del 02 de enero de $1973^{3}$ para proteger y asegurar el abastecimiento del embalse Los Laureles, uno de los principales acopios de agua para la capital.

\footnotetext{
${ }^{1}$ Coeficiente de Gini de 0.54 para 2013 y aproximadamente el $60 \%$ de los ingresos nacionales se encuentra distribuido entre el $20 \%$ de la población más rica y en el $20 \%$ de la población más pobre, se distribuye el $2.02 \%$ de esos ingresos nacionales. $(\mathrm{CIDH}, 2015)$

${ }^{2}$ Las dos principales ciudades del país figuraban entre las primeras ciudades más violentas del mundo en 2016. El MDC se encontró en la cuarta posición con una tasa de 85.09 homicidios por cada 10,000 habitantes. (SJyP, 2017).

${ }^{3}$ Datos obtenidos de la Modelación Hidrológica para la Sub cuenca de Río Guacerique por medio de la Herramienta ArcSwat bajo escenarios de cambios en el uso de suelos y precipitación realizada por el Instituto Hondureño de Ciencias de la Tierra (IHCIT)en 2013.
} 


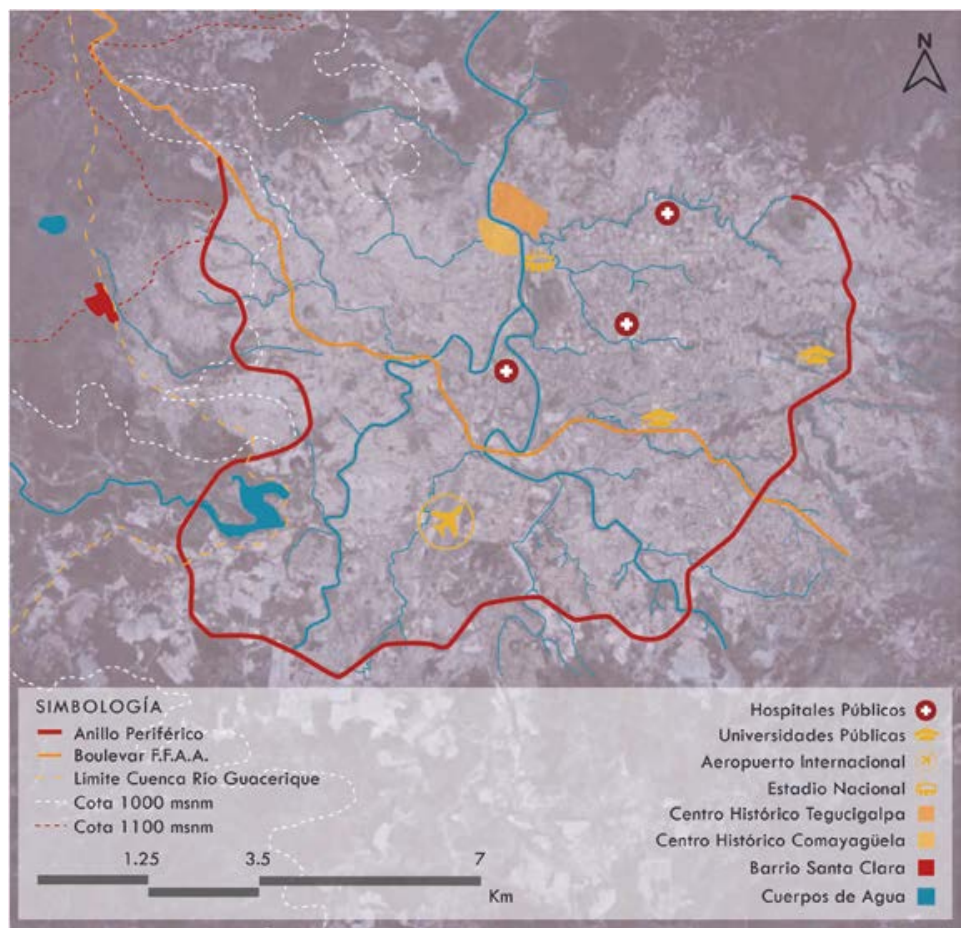

Fig. 01 Ubicación del barrio Santa Clara en el contexto municipal. Fuente: Elaboración propia en base a Google Earth (2018), y el IHCIT (2013).

Para 2018, Santa Clara era el único barrio en el área urbana donde TECHO-Honduras mantenía su apoyo desde el 2011. El barrio cuenta con 11 años de existencia y actualmente la mayor cantidad de personas (declaradas en las encuestas) no supera los 35 años de edad (42.26\% entre los 18 a 34 años y $39.13 \%$ menores de 18 años).

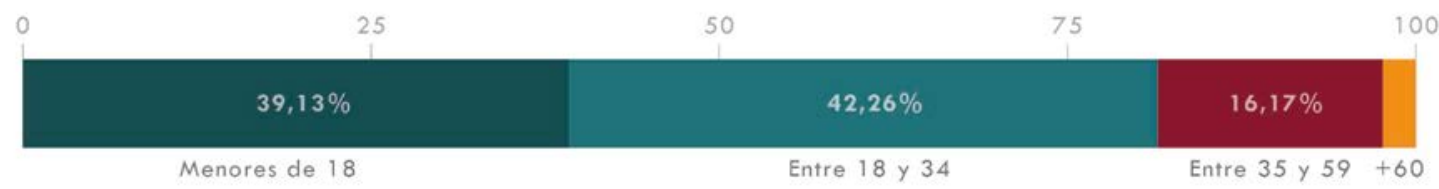

Fig. 02 Distribución de población por grupos de edades. Fuente: Elaboración propia en base a la encuesta, (2018).

El promedio declarado de ingresos fue de L. 1,450.00 (\$US 60.82 ${ }^{4}$ ), encontrándose este por debajo del costo promedio mensual de la canasta básica de alimentos per cápita. Estas familias, en términos de ingresos, se encuentran en condiciones de pobreza por percibir menos de \$US 2.00 diarios por persona.

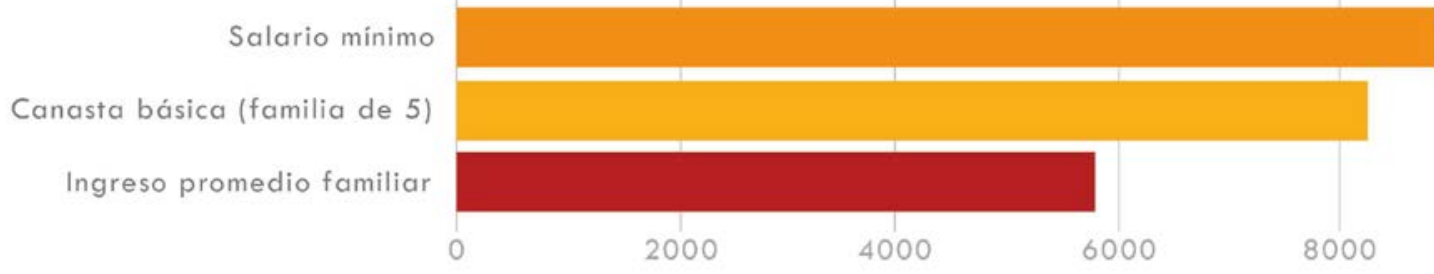

Fig. 03 Comparación de ingresos promedios mensuales con salario mínimo y canasta básica mensual per cápita. Fuente: Elaboración propia con la información de la encuesta (2018) y datos de la Secretaría de Trabajo y Seguridad Social, (2018).

\footnotetext{
${ }^{4}$ Tasa de cambio del 04 de mayo de 2018: $\$ 1.0=$ L. 23.8408
} 


\section{Resultados}

\subsection{Mercado informal del suelo y dinámicas de poder}

Un elemento que destaca en la complejidad del caso del barrio Santa Clara, se refleja en términos de la propiedad del suelo. El barrio surgió como una invasión, sin embargo poco tiempo después los habitantes llegaron a un acuerdo verbal con el presunto dueño de esos terrenos. La Gerencia de Acceso a la Tierra (GAT) de la Alcaldía Municipal del Distrito Central (AMDC) declaró en la entrevista que esas tierras forman parte del ejido municipal, a pesar de que el Patronato ${ }^{5}$ del barrio presentó frente al Servicio Autónomo Nacional de Acueductos y Alcantarillado (SANAA) un "Título Definitivo de Propiedad" con fecha de diciembre de 2001, otorgado por el Instituto Nacional Agrario (INA) a la persona con quien realizaron el acuerdo, acompañado de constancias del departamento de Catastro del AMDC con claves debidamente inscritas en el Instituto de la Propiedad.

Si bien no se conoce el contexto en el que estas tierras fueron transferidas, surge el cuestionamiento sobre los términos y condiciones en que esos terrenos llegaron a ser propiedad del INA, y posteriormente de este particular, pues esos terrenos pertenecen al área nombrada como "Zona Forestal Protegida del Patrimonio Público Forestal Inalienable" por el decreto No. 03-73 (aún vigente).

Alrededor del año 2016, algunos vecinos fueron informados de la posición de la GAT, y desde entonces ha existido incertidumbre sobre la validez de los pagos que ellos han estado realizando al particular que cuenta con el Título extendido por el INA. Algunos vecinos tomaron la decisión de no continuar pagando, y nuevos grupos de personas llegaron a conformar nuevos barrios en terrenos que la Junta Directiva del Patronato proclama como tierras del barrio Santa Clara.

Dicha Junta Directiva está a cargo del cobro de la cuota de pago que cada familia acordó con el dueño particular, y también exige una cuota por concepto de "afiliación", que se destina para proyectos de beneficio colectivo. A pesar de la incertidumbre generada en torno al tema de la propiedad de las tierras, esta Junta sigue realizando procesos de cobro y venta a nuevos habitantes, manteniendo un fuerte control sobre las deudas que cada familia ha adquirido. De este modo se ha evidenciado que existen intereses económicos más fuertes que los intereses colectivos y prueba de ello es que dentro de las advertencias que se extiende ante la falta de pagos se encuentra el desalojo.

Las dinámicas de poder existentes en el barrio se reflejan en el modo en que los vecinos entienden a la Junta Directica; un grupo cerrado que ejerce un fuerte poder de manera vertical con respecto al resto de los miembros de la comunidad, que administra el territorio y que ejerce un control fuertemente celado sobre los diferentes procesos que se realizan en el barrio. Durante las entrevistas también se reveló que esta Junta Directiva, que ha sido presidida por la misma persona desde 2009, no apoyó ni validó los procesos que realizaron tres Juntas de Aguas $^{6}$, dividiendo de este modo la opinión de los vecinos.

Ese tipo de situaciones reducen los niveles de confianza hacia las organizaciones internas y pueden mermar la participación activa de los diferentes miembros, debilitando el CSC. Al preguntar a las personas encuestadas sobre su disposición para colaborar con las diferentes organizaciones y actores que trabajan en el barrio, en una escala de 0 al 5 , donde 5 indica total confianza, fue hacia el patronato que expresaron menor confianza.

\footnotetext{
${ }^{5}$ Es la forma jurídica de asociación, de comunidades en un territorio determinado, reconocida por las instituciones hondureñas.

${ }^{6}$ Las Juntas de Aguas son los únicos organismos barriales reconocidos por el SANAA para todos los procesos relacionados al acceso a agua potable y saneamiento, y dicha institución promueve y exige que su conformación sea ajena a los patronatos (a pesar de que la AMDC reconoce la figura de patronato).
} 


\subsection{Capital Social Comunitario}

La privacidad fue el aspecto mejor valorado del barrio y refleja una actitud que se contrapone al interés por ser parte de una sociabilidad activa al interior del barrio. Con ello las redes sociales internas cuentan con vínculos débiles por lo que, las posibilidades de movilizar recursos a través de ellos para ampliar las oportunidades colectivas se ven disminuidas.

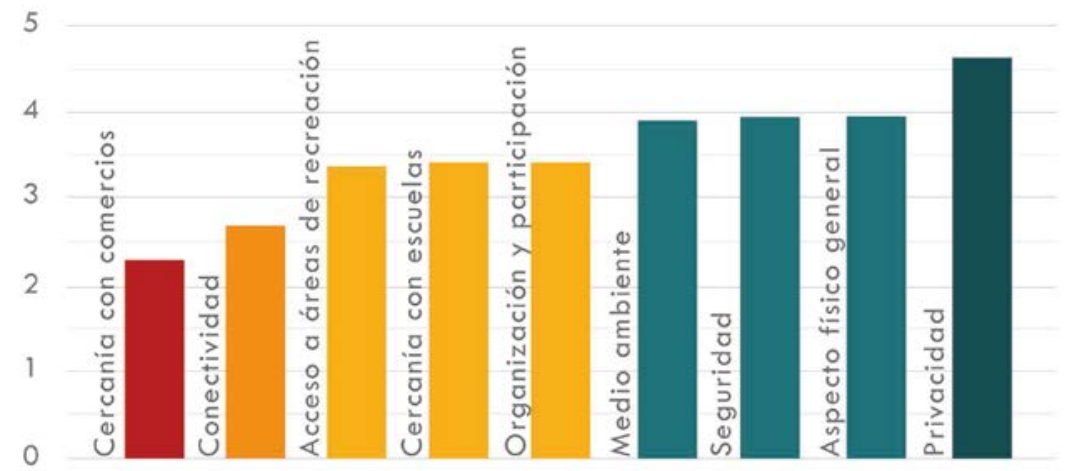

Fig. 04 Calificación del barrio en diferentes aspectos. Fuente: Elaboración propia a partir de encuesta (2018).

La cultura de violencia generalizada en las áreas urbanas y la estigmatización a la que se ven sometidos este tipo de barrios son factores que afectan la confianza entre vecinos, pues la latente inseguridad disminuye las expectativas que las personas pueden tener sobre los otros que le rodean si no forman parte de un círculo familiar. La desconfianza general dentro del barrio se evidencia en la poca frecuencia con la que los vecinos hacen uso de los espacios públicos del barrio y en el promedio declarado de personas que los participantes conocen de nombre y el promedio de amistades que declararon tener dentro del barrio: 20 personas conocidas y 4 amistades (en su mayoría familiares). En general, la confianza que las personas declararon tener hacia sus vecinos, en la escala de 0 a 5 , alcanzó un promedio de 2.53 .

Después de la privacidad, la mayor valoración del barrio estuvo en la seguridad (3.95 de 5). Esto se puede explicar con una "lógica seguritaria" que impregna los vínculos sociales (Lunecke, 2016), y la alta valoración de la privacidad revela además que la concepción de seguridad también se relaciona con poder permanecer excluidos en su espacio familiar, y hace referencia al como los individuos usan mecanismos de distanciamiento y permanecen en una esfera de consumo privatizado con las que se debilita la solidaridad (Bauman, 2004 citado por Lunecke, 2016).

Al perderse el interés o la confianza en un proyecto (o en sus dirigentes como ha sucedido en Santa Clara), aquellos que dejan de participar, más allá de realizar un pago para acceder al beneficio del proyecto (en caso de que el mismo se concrete), no verán más la necesidad de cooperar y tampoco sentirán la obligación de ejercer reciprocidad, a menos que existan intereses individuales. Tras la investigación se encontró que un ejemplo de lo antes mencionado es el proceso de construcción de viviendas que TECHO realizó. Si bien es cierto que existieron proyectos de beneficio colectivo, las 64 viviendas construidas por la organización (de 263 existentes para 2016), representaron beneficios a escala familiar, y muchos vecinos participaron con la aspiración de recibir posteriormente dicho beneficio.

En el barrio se encontró una baja frecuencia en la realización de actividades de socialización y de acciones o actitudes que impliquen la movilización de recursos motivados por la confianza; deportes o juegos colectivos, solicitudes de diferentes tipos de colaboraciones y realización de favores como se observa en la siguiente figura: 


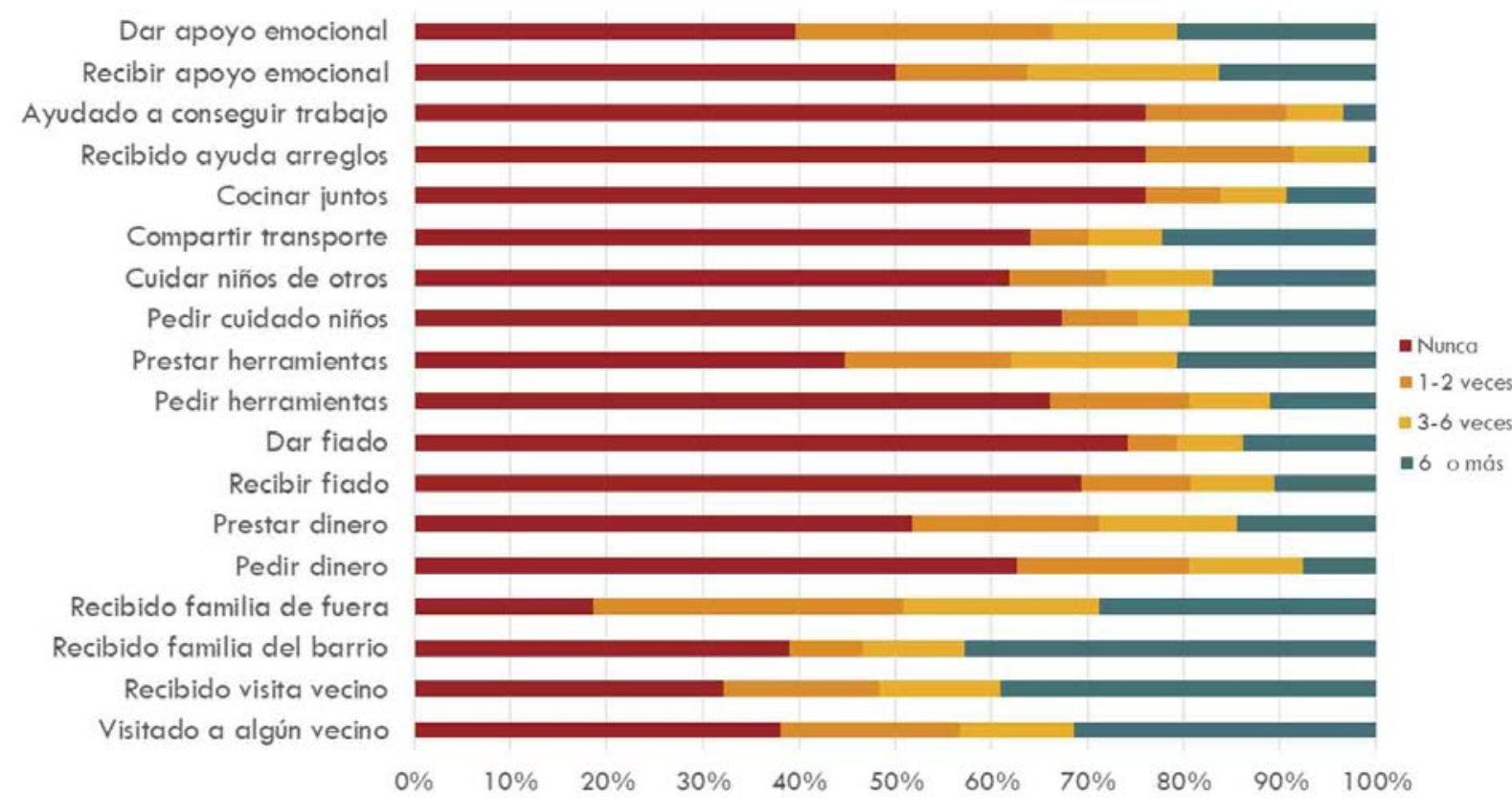

Fig. 05 Promedios de frecuencia de actividades colaborativas realizadas en los últimos 6 meses. Fuente: Elaboración propia a partir de encuesta (2018).

\subsection{Divergencias institucionales y acceso a servicios básicos}

Existen diferentes modos de entender a los barrios informales, y la incongruencia entre dichas concepciones, alerta sobre la falta de articulación entre las diferentes estrategias de intervención institucional.

EI SANAA los llama "barrios en desarrollo" considerando que, esos barrios que inician de manera informal, logran desarrollarse al adquirir los servicios con el esfuerzo organizado de la comunidad.

La Empresa Nacional de Energía Eléctrica (ENEE) los llama barrios (o zonas) "urbano-marginales" o "crecimientos periféricos" y los entiende como aquellos barrios que se encuentran fuera del "casco urbano normal", sin un desarrollo urbanístico y que no cuentan con servicios básicos.

La GAT los llama "asentamientos humanos" y equipara ese concepto con el término de "invasiones", con lo que le da una connotación negativa asociada a dicho modo de apropiación de la tierra.

TECHO, para efectos del Censo de Asentamientos Informales, los entiende similar a lo que expone Antillano (2005); en términos de las carencias que ahí existen, pues consideran informal a aquel barrio donde no exista tenencia segura de la tierra y/o no exista acceso a dos de los siguientes servicios básicos: agua potable, saneamiento y energía eléctrica.

Los procesos burocráticos que exigen estas instituciones (a excepción de TECHO) suelen traer complicaciones. Para acceder a la red de energía eléctrica, la ENEE exige una constancia de la AMDC que indique que las tierras no se encuentran en riesgo (ni en pleno desarrollo) de un litigio. Para contar con dicha constancia, Santa Clara tendría que pasar primero por un proceso de investigación que aclare frente a la GAT la propiedad de los terrenos. Sin embargo, la persona entrevistada de la GAT reconoció tener conocimiento de que en la mayoría de los casos, los barrios informales logran obtener primero los servicios urbanos para posteriormente exigir los títulos de propiedad.

En cuanto al agua potable y el saneamiento, el barrio cuenta con una condicionante que no le permite acceder a los servicios, debido a que se ubica sobre la cota máxima de capacidad de abastecimiento de la red formal 
del SANAA, por lo que la única opción es el abastecimiento por acarreo. Para ello, en 2014 tuvieron apoyo de un departamento del SANAA para el diseño y construcción de un tanque elevado donde almacenar el agua. El tanque fue un logro concretado pero no está en funcionamiento debido a que el departamento responsable del abastecimiento, no aceptó acarrear el agua por no contar con la capacidad de desarrollar un proyecto integral que pueda incluir el control sobre el saneamiento y asegurar así la protección de la sub-cuenca en la que se encuentran ubicados.

Ante estos obstáculos la alternativa que tienen en época seca, es comprar el agua a carros cisternas privados. Todas las personas encuestadas compran el agua a un costo de L. 29.00 (\$US 1.22) por barril de 42 galones $\left(0.16 \mathrm{~m}^{3}\right.$ ) llegando a pagar L. 181.25 (\$US 7.60) por $\mathrm{m}^{3}$. Para acceder a lo mínimo recomendado para un mes por la Organización Mundial de la Salud, una familia de 5 miembros (promedio del barrio) tendrían que invertir L. 2,718.75 (\$US 114.04) lo que representa un 333\% más de lo que pagaría por el mismo consumo una familia de igual cantidad de miembros que habita en un barrio céntrico y recibe el agua directo de la red del SANAA. ${ }^{7}$

En cuanto a la energía eléctrica, la Junta Directiva del patronato realizó gestiones de manera independiente, con un agente privado que invirtió en la comunidad, proveyéndoles de la infraestructura necesaria. La comunidad deberá pagar por esta infraestructura en cuotas y eventualmente tendrán que asumir una deuda adquirida con la ENEE por el consumo de energía promedio que hayan tenido sin estar previamente autorizados por ellos.

Para necesidades como el saneamiento y el mejoramiento de vías, la comunidad ha optado por aprovechar oportunidades de carácter clientelar; el patronato ha realizado gestiones con funcionarios de la AMDC para el proyecto de pavimentación de la calle principal, y muchas familias de forma individual realizan gestiones con los "Guías de Familia", que representan a la Secretaría de Desarrollo e Inclusión Social (SEDIS) en cada barrio para distribuir beneficios asistenciales como letrinas, láminas y pisos de concreto que son promovidos por el gobierno de turno.

\section{Conclusiones}

El Capital Social Comunitario es débil en la comunidad de Santa Clara. A pesar de que existen vínculos familiares entre los miembros que pueden propiciar la reciprocidad y la cooperación, existen condiciones y dificultades en el desarrollo de proyectos colectivos que debilitan las dinámicas sociales internas y disminuyen la frecuencia con que se observan "comportamientos confiables".

Los vínculos con los que cuenta cada miembro de la comunidad no representan un soporte social para consolidar una red colectiva, al contrario, la mayoría de los miembros prefieren mantener una vida en privado y abstenerse de socializar dentro del barrio. Para acceder a proyectos de infraestructura básica, los recursos han sido movilizados por actores que mantienen una gran cuota de poder y son quienes tienen los recursos para realizar conexiones con agentes externos. Si estos actores desaparecen de la comunidad, esa capacidad de establecer vínculos a lo externo del barrio se pierde con ellos. De hecho, no se puede afirmar que el barrio es producto de acciones colectivas, pues las motivaciones más fuertes para asentarse en el barrio se ven relacionadas a las posibilidades que oferta ese mercado informal de suelo.

Las dinámicas institucionales de intervención a escala familiar, no representan beneficios en términos de participación y fortalecimiento de la organización y cooperación comunitaria. No se encontró una relación entre

\footnotetext{
${ }^{7}$ Una familia de 5 personas requiere de 15 metros cúbicos (15,000 litros) de agua para alcanzar el promedio de consumo de agua recomendado por la OMS según Howard, G. \& Bartram J. (2003), en un mes. Se multiplicó esa cantidad por la tarifa que cobra el SANAA en la Residencial Tres Caminos (L.41.80 = \$US 1.75), incluyendo el pago por derecho a conexión a la red. El costo del servicio con esas condiciones es de L. $627.00=\$$ US 26.3.
} 
la participación y el hecho de pertenecer a una familia que haya sido beneficiada por algún programa de beneficio individual, como la vivienda de TECHO o los impulsados por la SEDIS. El primer caso refleja lo que Durston (2005) Ilama "semiclientelismo", y el segundo representa un "clientelismo pasivo" que crea dependencia al esperar que las personas sean receptores pasivos de sus productos. Entre los procesos institucionales del gobierno local y central, existen incongruencias que condicionan el verdadero aprovechamiento de los programas que se ejecutan, y dejan en evidencia que no existe una estrategia conjunta para el mejoramiento de estos barrios ni de las condiciones de pobreza que imperan en nuestra sociedad.

Si bien en el caso de Santa Clara, el barrio ha logrado dotarse de algunos beneficios, las acciones que los promovieron no tuvieron como objetivo final consolidar el barrio en la ciudad formal, dar reconocimiento a la comunidad y tampoco se ven relacionados al fortalecimiento de CSC, pues las dinámicas de poder y las condiciones de vulnerabilidad han repercutido directamente en la construcción de dicho capital.

\section{BIBLIOGRAFÍA}

A. $\quad$ DE LOMNITZ, L. (1975). Cómo sobreviven los marginados. México: Siglo XXI Editores.

ANTILLANO, A. (2005). La lucha por el reconocimiento y la inclusión en los barrios populares: la experiencia de los Comités de Tierras Urbanas. Revista Venezolana de Economía y Ciencias Sociales v. 11 No. 3, (págs. 205-218).

ATRIA, R. (2003). Capital Social: Concepto, dimensiones y estrategias para su desarrollo. En R. Atria, M. Siles, I. Arriagada, L. Robison, S. Whiteford (Compiladores), Capital social y reducción de la pobreza en América Latina y el Caribe: en busca de un nuevo paradigma (págs. 581-590). Santiago de Chile: Publicación de las Naciones Unidas.

BOURDIEU, P. (1986). The forms of capital. En J. Richardson, Handbook of Theory and Research for the Sociology of Education (págs. 241-258). New York: Greenwood.

CABALLERO, E., (2010). Planificación del Territorio Urbano en Honduras: Entre la Acción Pública y el Mercado. Revista Postgrados UNAH, No. 4 Vol. 1, (págs. 68-79).

CASTELLS, M., (1987). Capital Multinacional, Estados Nacionales, Comunidades Locales. . México: Siglo XXI Editores.

CRAVINO, M. (2006). Las villas de la ciudad. Mercado e informalidad urbana. Buenos Aires: Universidad Nacional de General Sarmiento.

DURSTON, J, (1999). Construyendo Capital Social Comunitario. Revista de la Cepal No. 69, (págs. 103-118).

DURSTON, J. (2000). ¿Qué es el capital social comunitario? Santiago de Chile: Serie Políticas Sociales, Comisión Económica para América Latina y el Caribe (CEPAL).

DURSTON, J., (2003). Capital social: parte del problema, parte de la solución, su papel en la persistencia y en la superación de la pobreza en América Latina y El Caribe. En R. Atria, M. Siles, I. Arriagada, L. Robison, S. Whiteford (Compiladores), Capital social y reducción de la pobreza en América Latina y el Caribe: en busca de un nuevo paradigma (págs. 147-202). Santiago de Chile: Publicación de las Naciones Unidas.

DURSTON, J. (2005). Superación de la pobreza, capital social y clientelismos locales. . En I. Arriagada, Aprender de la experiencia: el capital social en la superación de la pobreza (págs. 47-58). Santiago de Chile: Publicaciones de las Naciones Unidas. 
GUTIÉRREZ, A. (2008). Redes e intercambio de capitales en condicones de pobreza: dimensión relacional y dimensión vincular. Redes. Revista Hispana para el Análisis de Redes Sociales, No. 14.

LUNECKE, A. (2016). Inseguridad ciudadana y diferenciación social en el nivel microbarrial: el caso del sector Santo Tomás, Santiago de Chile. Revista Eure, Vol. 42, No. 125, (págs. 109-129).

PINO, A. \& OJEDA, L. (2013). Ciudad y Hábitat Informal: Las tomas de terreno y la autoconstrucción en las quebradas de Valparaíso. Revista INVI No. 78, Vol. 28, (págs. 109-140).

PÍREZ, P. (2013). Los Servicios Urbanos en América Latina. . En B. Ramírez \& E. Pradilla, Teorías sobre la ciudad en América Latina, Volumen II (págs. 455-504). México: Universidad Autónoma Metropolitana y Editores e Impresores Profesionales Edimpro.

RACZYNSKI, D. \& SERRANO, C. (2005). Programas de superación de la pobreza y el capital social. Evidencias y aprendizajes de la experiencia en Chile. . En I. Arriagada, Aprender de la experiencia: el capital social en la superación de la pobreza (págs. 99-132). Santiago de Chile: Publicaciones de las Naciones Unidas.

TIRONI, M. (2003). Nueva pobreza urbana. Vivienda y capital social en Santiago de Chile, 1985-2001. Santiago de Chile: Universidad de Chile, Predes/RIL Editores.

TRUNDLE, L. (2012). De las "encomiendas" al mercado. Quinientos años de desigualdad. En C. C. Hábitat, Derecho al suelo y la ciudad en América Latina; La realidad y los caminos posibles (págs. 155-166). Montevideo: Ediciones Trilce.

\section{Fuentes electrónicas}

COMISIÓN INTERAMERICANA DE DERECHOS HUMANOS [CIDH], (2015). Situación de los Derechos Humanos en Honduras. http://www.oas.org/es/cidh/informes/pdfs/honduras-es-2015.pdf (Consulta:08/03/2019)

HOWARD, G. \& BARTRAM, J. (2003). Domestic Water Quantity, Service Level and Health. World Health Organization.: http://www.who.int/water sanitation health/diseases/WSH0302.pdf (Consulta:08/03/2019)

INSTITURO NACIONAL DE ESTADÍSTICA [INE], (2016). Hogares en condición de pobreza 2016. http://www.ine.gob.hn/index.php/25-publicaciones-ine/91-linea-de-pobreza.html (Consulta:08/03/2019)

LEFEBVRE, H. (1970). La revolución urbana. https://leerlaciudadblog.files.wordpress.com/2016/05/lefebvrela-revolucic3b3n-urbana.pdf (Consulta:08/03/2019) 